Mathematical Research Letters 4, 69-78 (1997)

\title{
SHIFTED JACK POLYNOMIALS, BINOMIAL FORMULA, AND APPLICATIONS
}

\section{A. Okounkov and G. Olshanski}

\begin{abstract}
In this note we prove an explicit binomial formula for Jack polynomials and discuss some applications of it.
\end{abstract}

\section{Jack polynomials $([\mathrm{M}, \mathrm{St}])$}

In this note we use the parameter

$$
\theta=1 / \alpha
$$

inverse to the standard parameter $\alpha$ for Jack polynomials. Jack symmetric polynomials $P_{\lambda}\left(x_{1}, \ldots, x_{n} ; \theta\right)$ are eigenfunctions of Sekiguchi differential operators

$$
\begin{aligned}
& D(u ; \theta)=V(x)^{-1} \operatorname{det}\left[x_{i}^{n-j}\left(x_{i} \frac{\partial}{\partial x_{i}}+(n-j) \theta+u\right)\right]_{1 \leq i, j \leq n}, \\
& D(u ; \theta) P_{\lambda}(x ; \theta)=\left(\prod_{i}\left(\lambda_{i}+(n-i) \theta+u\right)\right) P_{\lambda}(x ; \theta),
\end{aligned}
$$

where $V(x)=\prod_{i<j}\left(x_{i}-x_{j}\right)$ is the Vandermonde determinant and $u$ is a parameter. The operators $\{D(u ; \theta), u \in \mathbb{C}\}$ generate a commutative algebra; denote it by $\mathfrak{D}(n ; \theta)$.

We normalize $P_{\lambda}\left(x_{1}, \ldots, x_{n} ; \theta\right)$ so that

$$
P_{\lambda}\left(x_{1}, \ldots, x_{n} ; \theta\right)=x_{1}^{\lambda_{1}} \ldots x_{n}^{\lambda_{n}}+\ldots,
$$

where dots stand for lower monomials in lexicographic order. Then one has

$$
P_{\left(\lambda_{1}+1, \ldots, \lambda_{n}+1\right)}(x ; \theta)=\left(\prod x_{i}\right) P_{\lambda}(x ; \theta) .
$$

Using (1.2) one can define Jack rational functions for any integers $\lambda_{1} \geq \cdots \geq \lambda_{n}$ and still (1.1), and hence the binomial theorem below, will hold.

Note that the eigenvalues in (1.1) are symmetric in variables $\lambda_{i}-\theta i, i=$ $1, \ldots, n$.

Received August 23, 1996.

The authors were supported by the Russian Basic Research Foundation (grant 95-01-00814). The first author was supported by the NSF grant DMS 9304580. 


\section{Shifted Jack polynomials ([S1,OO,Ok1,KS,Ok3])}

Denote by $\Lambda^{\theta}(n)$ the algebra of polynomials $f\left(x_{1}, \ldots, x_{n}\right)$ that are symmetric in variables $x_{i}-\theta i$. We call such polynomials shifted symmetric. By (1.1) one has the Harish-Chandra isomorphism

$$
\mathfrak{D}(n ; \theta) \rightarrow \Lambda^{\theta}(n)
$$

which takes an operator $D \in \mathfrak{D}(n ; \theta)$ to the polynomial $d \in \Lambda^{\theta}(n)$ such that

$$
D P_{\lambda}(x ; \theta)=d(\lambda) P_{\lambda}(x ; \theta) \text {. }
$$

If $D$ is of order $k$ then $\operatorname{deg} d=k$.

Let $\mu$ be a partition (also viewed as a diagram). Recall that for a square $s=(i, j) \in \mu$ the numbers

$$
\begin{array}{ll}
a(s)=\mu_{i}-j, & a^{\prime}(s)=j-1, \\
l(s)=\mu_{j}^{\prime}-i, & l^{\prime}(s)=i-1,
\end{array}
$$

are called arm-length, arm-colength, leg-length, and leg-colength respectively. Set

$$
H(\mu)=\prod_{s \in \mu}(a(s)+\theta l(s)+1)
$$

According to a general result of S. Sahi [S1] there exists the unique polynomial $P_{\mu}^{*}(x ; \theta)$ in $\Lambda^{\theta}(n)$ such that $\operatorname{deg} P_{\mu}^{*} \leq|\mu|$ and

$$
P_{\mu}^{*}(\lambda ; \theta)= \begin{cases}H(\mu), & \lambda=\mu \\ 0, & |\mu| \leq|\lambda|, \mu \neq \lambda\end{cases}
$$

Here we assume $\mu$ and $\lambda$ have length $\leq n$. It is clear that the uniqueness of $P_{\mu}^{*}(x, \theta)$ follows easily from its existence.

The polynomials $P_{\mu}^{*}(x ; 1)$ were studied in $[\mathrm{OO}]$ and $[\mathrm{Ok} 1]$. They are closely related to Schur polynomials and are called shifted Schur functions. In particular, the Schur function $s_{\mu}$ is the highest degree term of $P_{\mu}^{*}(x ; 1)$. Shifted Schur functions have a very rich combinatorics and numerous applications (such as Capelli-type identities or asymptotic character theory, see [OO,Ok1,N,Ok2] and references therein).

For general $\theta$, F. Knop and S. Sahi proved (see $[\mathrm{KS}]$, Theorem 5.2) that

$$
P_{\mu}^{*}(\lambda ; \theta)=0, \quad \text { unless } \quad \mu \subset \lambda,
$$

and that again $([\mathrm{KS}]$, Corollary 4.7)

$$
P_{\mu}^{*}(x ; \theta)=P_{\mu}(x ; \theta)+\quad \text { lower degree terms. }
$$


Their proof was based on difference equations for the polynomial $P_{\mu}^{*}(x ; \theta)$. For $\theta=1$ these properties follow immediately from explicit formulas for $P_{\mu}^{*}(x ; 1)$.

Explicit formulas for the polynomials $P_{\mu}^{*}(x ; \theta)$, which generalize explicit formulas for shifted Schur functions, were found in [Ok3] (see, for example, (2.4) below). In particular, they provide an effective proof of the existence of $P_{\mu}^{*}(x ; \theta)$ and a different proof of (2.2) and (2.3).

We call these polynomials shifted Jack polynomials. They are a degenerate case of shifted Macdonald polynomials [Kn,S2,Ok3].

It easily follows from the definition that $P_{\mu}^{*}(x ; \theta)$ are stable, that is

$$
P_{\mu}^{*}\left(x_{1}, \ldots, x_{n}, 0 ; \theta\right)=P_{\mu}^{*}\left(x_{1}, \ldots, x_{n} ; \theta\right)
$$

provided $\mu$ has at most $n$ parts. Hence, one can consider shifted Jack polynomials in infinitely many variables.

The following combinatorial formula for shifted Jack polynomials was proved in [Ok3]. Let us call a tableau $T$ on $\mu$ a reverse tableau if its entries strictly decrease down the columns and weakly decrease in the rows. By $T(s)$ denote the entry in the square $s \in \mu$. We have

$$
P_{\mu}^{*}(x ; \theta)=\sum_{T} \psi_{T}(\theta) \prod_{s \in \mu}\left(x_{T(s)}-a^{\prime}(s)+\theta l^{\prime}(s)\right),
$$

where the sum is over all reverse tableaux on $\mu$ with entries in $\{1,2, \ldots\}$ and $\psi_{T}(\theta)$ is the same $\theta$-weight of a tableau that enters the combinatorial formula for ordinary Jack polynomials (see [St] or [M],(VI.10.12))

$$
P_{\mu}(x ; \theta)=\sum_{T} \psi_{T}(\theta) \prod_{s \in \mu} x_{T(s)} .
$$

The coefficients $\psi_{T}(\theta)$ are rational functions of $\theta$.

\section{Binomial formula}

Given a partition $\mu$ and a number $t$ set

$$
\begin{aligned}
H^{\prime}(\mu) & =\prod_{s \in \mu}(a(s)+\theta l(s)+\theta), \\
(t)_{\mu} & =\prod_{s \in \mu}\left(t+a^{\prime}(s)-\theta l^{\prime}(s)\right) .
\end{aligned}
$$

If $\mu=(m)$ then $(t)_{\mu}$ is the standard shifted factorial. We have (see [M], VI.10.20)

$$
P_{\mu}(1, \ldots, 1 ; \theta)=(n \theta)_{\mu} / H^{\prime}(\mu) .
$$

Set (see [M], VI.10.16)

$$
Q_{\mu}(x ; \theta)=\frac{H^{\prime}(\mu)}{H(\mu)} P_{\mu}(x ; \theta) .
$$

The main result of this note is the following 


\section{Theorem.}

$$
\frac{P_{\lambda}\left(1+x_{1}, \ldots, 1+x_{n} ; \theta\right)}{P_{\lambda}(1, \ldots, 1 ; \theta)}=\sum_{\mu} \frac{P_{\mu}^{*}\left(\lambda_{1}, \ldots, \lambda_{n} ; \theta\right) Q_{\mu}\left(x_{1}, \ldots, x_{n} ; \theta\right)}{(n \theta)_{\mu}} .
$$

Note that by virtue of (2.2) the summation in the binomial formula (3.2) is only over such $\mu$ that

$$
\mu \subset \lambda
$$

Let $\mathcal{O}_{1}$ be the local ring of symmetric rational functions regular at the point

$$
\overline{1}=(1, \ldots, 1)
$$

and let $\mathfrak{m}_{1} \subset \mathcal{O}_{1}$ be its maximal ideal. We call functionals

$$
\psi \in\left(\mathcal{O}_{1} / \mathfrak{m}_{1}^{k}\right)^{*} \subset \mathcal{O}_{1}^{*}
$$

symmetric distributions of order $\leq k$ supported at the point $\overline{1}$ defined in (3.3). Denote by $\mathcal{S}_{1}$ the space of all symmetric distributions supported at $\overline{1}$. Looking at the highest derivatives one easily proves that the map

$$
\mathfrak{D}(n ; \theta) \rightarrow \mathcal{S}_{1}
$$

which takes an operator $D$ to the distribution $\psi_{D}$

$$
\psi_{D}(f)=(D f)(1, \ldots, 1)
$$

is an order preserving isomorphism of linear spaces.

Proof of the theorem. Consider the Taylor expansion of $P_{\lambda}(x ; \theta)$ about the point $\overline{1}$. Since Jack polynomials form a linear basis in symmetric polynomials this expansion has the form

$$
P_{\lambda}\left(1+x_{1}, \ldots, 1+x_{n} ; \theta\right)=\sum_{\mu} \psi_{\mu}\left(P_{\lambda}\right) P_{\mu}(x ; \theta), \quad \psi_{\mu} \in \mathcal{S}_{1}
$$

Note that $\psi_{\mu}$ is of order $\leq|\mu|$. By virtue of the isomorphisms (2.1) and (3.4) we have

$$
P_{\lambda}\left(1+x_{1}, \ldots, 1+x_{n} ; \theta\right)=P_{\lambda}(1, \ldots, 1) \sum_{\mu} f_{\mu}(\lambda) P_{\mu}(x ; \theta), \quad f_{\mu} \in \Lambda^{\theta}(n)
$$

where $\operatorname{deg} f_{\mu} \leq|\mu|$. On the other hand

$$
P_{\lambda}\left(1+x_{1}, \ldots, 1+x_{n} ; \theta\right)=P_{\lambda}\left(x_{1}, \ldots, x_{n} ; \theta\right)+\text { lower degree terms. }
$$


From (3.6) and (3.7) we obtain

$$
f_{\mu}(\lambda)= \begin{cases}0, & |\lambda| \leq|\mu|, \lambda \neq \mu \\ 1 / P_{\mu}(1, \ldots, 1), & \lambda=\mu\end{cases}
$$

Since $f_{\mu}$ is a polynomial of degree $\leq|\mu|$ it is completely determined by its values at the points $\lambda,|\lambda| \leq|\mu|$. Therefore, $f_{\mu}$ is proportional to $P_{\mu}^{*}$ and by $(3.1)$

$$
f_{\mu}(x)=\frac{H^{\prime}(\mu)}{(n \theta)_{\mu} H(\mu)} P_{\mu}^{*}(x ; \theta),
$$

which concludes the proof.

Remark. The coefficients $\left(\begin{array}{l}\lambda \\ \mu\end{array}\right)_{\theta}$ in the expansion

$$
\frac{P_{\lambda}\left(1+x_{1}, \ldots, 1+x_{n} ; \theta\right)}{P_{\lambda}(1, \ldots, 1 ; \theta)}=\sum_{\mu}\left(\begin{array}{l}
\lambda \\
\mu
\end{array}\right)_{\theta} \frac{P_{\mu}\left(x_{1}, \ldots, x_{n} ; \theta\right)}{P_{\mu}(1, \ldots, 1 ; \theta)} .
$$

are called the generalized binomial coefficients. They were studied by C. Bingham $[\mathrm{Bi}]$ in the special case $\theta=1 / 2$, using group-theoretical methods, and by M. Lassalle [La] for general $\theta$. Then a proof of the main results announced in [La] was proposed by J. Kaneko (see [K], section 5). Our theorem says that this binomial coefficient

$$
\left(\begin{array}{l}
\lambda \\
\mu
\end{array}\right)_{\theta}=\frac{P_{\mu}^{*}(\lambda ; \theta)}{H(\mu)}
$$

is a shifted symmetric polynomial in $\lambda$ which by (2.4) and (2.5) is just as complex as the Jack polynomial $P_{\mu}(x ; \theta)$ itself. The main results of Lassalle can be easily deduced from (3.8). For example, it is clear that (3.8) does not depend on $n$ and vanishes unless $\mu \subset \lambda$. The recurrence relation (see [La], théorèmes $2,3,5$ and corollaire in section 6 ) is equivalent to the formula (5.2) below.

Note also that the coefficients (3.8) admit a simple formula when $\theta=1$ (see A. Lascoux [Lasc] and Example 10 in [M], section I.3) or when $\lambda_{1}=\cdots=\lambda_{n}$ (see J. Faraut and A. Korányi [FK], Prop. XII.1.3 (ii)).

Binomial formulas for characters of classical groups are discussed in [OO2 $]^{1}$.

\section{Bessel functions $([\mathrm{D}, \mathrm{Op}, \mathrm{J}])$}

For a real vector $l=\left(l_{1}, \ldots, l_{n}\right)$ denote by

$$
[l]=\left(\left[l_{1}\right], \ldots,\left[l_{n}\right]\right)
$$

its integral part. Suppose that $l_{1} \geq \cdots \geq l_{n}$. By definition, put

$$
F(l, x ; \theta)=\lim _{\kappa \rightarrow \infty} \frac{P_{[\kappa l]}\left(1+x_{1} / \kappa, \ldots, 1+x_{n} / \kappa ; \theta\right)}{P_{[\kappa l]}(1, \ldots, 1 ; \theta)} .
$$

From (3.2) and (2.3) we have

\footnotetext{
${ }^{1}$ Recently the first author found a binomial formula for Macdonald polynomials (both shifted and ordinary) and also for Koornwinder polynomials, see [Ok4-5].
} 
Proposition.

$$
F(l, x ; \theta)=\sum_{\mu} \frac{P_{\mu}\left(l_{1}, \ldots, l_{n} ; \theta\right) Q_{\mu}\left(x_{1}, \ldots, x_{n} ; \theta\right)}{(n \theta)_{\mu}} .
$$

Proof. We have (one easily checks absolute convergence of all series)

$$
\begin{aligned}
F(l, x ; \theta) & =\lim _{\kappa \rightarrow \infty} \frac{P_{[\kappa l]}\left(1+x_{1} / \kappa, \ldots, 1+x_{n} / \kappa ; \theta\right)}{P_{[\kappa l]}(1, \ldots, 1 ; \theta)} \\
& =\sum_{\mu} \lim _{\kappa \rightarrow \infty} \frac{P_{\mu}^{*}\left(\left[\kappa l_{1}\right], \ldots,\left[\kappa l_{n}\right] ; \theta\right) Q_{\mu}\left(x_{1} / \kappa, \ldots, x_{n} / \kappa ; \theta\right)}{(n \theta)_{\mu}} \\
& =\sum_{\mu} \lim _{\kappa \rightarrow \infty} \frac{\kappa^{-|\mu|} P_{\mu}^{*}\left(\left[\kappa l_{1}\right], \ldots,\left[\kappa l_{n}\right] ; \theta\right) Q_{\mu}\left(x_{1}, \ldots, x_{n} ; \theta\right)}{(n \theta)_{\mu}} \\
& =\sum_{\mu} \frac{P_{\mu}\left(l_{1}, \ldots, l_{n} ; \theta\right) Q_{\mu}\left(x_{1}, \ldots, x_{n} ; \theta\right)}{(n \theta)_{\mu}}
\end{aligned}
$$

where the second equality is based on the binomial formula and the last equality follows from (2.3).

We call $F(l, x ; \theta)$ the Bessel functions. They are in the same relation to Jack polynomials as ordinary Bessel functions to Jacobi polynomials. They are eigenfunctions of the corresponding degeneration of Sekiguchi operators. The formula (4.2) makes obvious the following known symmetry

$$
F(l, x ; \theta)=F(x, l ; \theta) .
$$

Let $H(n, \mathbb{R})$ denote the space of real symmetric matrices of order $n$, let $X, Y \in$ $H(n, \mathbb{R})$, and let $x=\left(x_{1}, \ldots, x_{n}\right)$ and $y=\left(y_{1}, \ldots, y_{n}\right)$ be the spectra of $X$ and $Y$. The compact orthogonal group $O(n)$ acts on the space $H(n, \mathbb{R})$ by conjugations. One can prove that

$$
F(y, x ; 1 / 2)=\int_{O(n)} \exp \left(\operatorname{tr}\left(Y u X u^{-1}\right)\right) d u,
$$

where $d u$ is the normalized Haar measure on $O(n)$. (Idea of proof: the normalized polynomials

$$
P_{\lambda}\left(z_{1}, \ldots, z_{n} ; 1 / 2\right) / P_{\lambda}(1, \ldots, 1 ; 1 / 2)
$$

are the spherical functions on the compact symmetric space $U(n) / O(n)$ while the integrals (4.3) are essentially the spherical functions on the associated Euclidean type symmetric space $O(n) \ltimes H(n, \mathbb{R})$. It is well-known (see $[\mathrm{DR}])$ that the spherical functions of Euclidean type can be obtained from the spherical functions of compact type by the limit transition (4.1).)

A similar interpretation of the functions $F(y, x ; \theta)$ also exists for $\theta=1$ and $\theta=2$. Then one has to consider orbits in the spaces $H(n, \mathbb{C})$ and $H(n, \mathbb{H})$ of complex and quaternionic Hermitian matrices, respectively. An application of the expansion (4.2) for $\theta=1$ is given in [OV]; similar results also hold for $\theta=1 / 2$ and $\theta=2$. 


\section{Formula for $\theta$-dimension of a skew diagram $\lambda / \mu$}

Define the $\theta$-dimension $\theta$-dim $\lambda / \mu$ of a skew diagram $\lambda / \mu$ as the following coefficient

$$
\left(\sum x_{i}\right)^{k} P_{\mu}(x ; \theta)=\sum_{|\lambda|=|\mu|+k} \theta-\operatorname{dim} \lambda / \mu P_{\lambda}(x ; \theta), \quad k=1,2, \ldots
$$

If $\theta=1$ then $\theta$-dim $\lambda / \mu$ equals the number of the standard tableaux on $\lambda / \mu$; for general $\theta$ each tableau $T$ is counted with a certain weight $\psi_{T}^{\prime}(\theta)$ given in $[\mathrm{M}]$, section VI.6. In particular, if $\mu=\emptyset$ then (see (5.6) below for a proof using shifted Jack polynomials)

$$
\theta-\operatorname{dim} \lambda=|\lambda| ! / H(\lambda)
$$

We have

\section{Proposition.}

$$
\frac{\theta-\operatorname{dim} \lambda / \mu}{\theta-\operatorname{dim} \lambda}=\frac{P_{\mu}^{*}(\lambda ; \theta)}{|\lambda|(|\lambda|-1) \cdots(|\lambda|-|\mu|+1)} .
$$

This proposition can be deduced from (3.8) and [La,K] (see the remark above), but it easier to give a direct proof, which uses the very same argument as in $[\mathrm{OO}]$, section 9 .

First one proves that

\section{Lemma.}

$$
\left(\sum x_{i}-|\mu|\right) P_{\mu}^{*}(x ; \theta)=\sum_{|\lambda|=|\mu|+1} \theta-\operatorname{dim} \lambda / \mu P_{\lambda}^{*}(x ; \theta) .
$$

Proof. Let $f$ be the difference of the LHS and RHS in (5.3). By (2.3) and (5.1) we have $\operatorname{deg} f \leq|\mu|$. One easily checks that $f(\nu)=0$ for all $|\nu| \leq|\mu|$. It follows that $f=0$.

Applying this lemma $k$ times, where $k=|\lambda|-|\mu|$, we obtain

$$
\left(\sum x_{i}-|\mu|\right) \cdots\left(\sum x_{i}-|\mu|-k+1\right) P_{\mu}^{*}(x ; \theta)=\sum_{|\nu|=|\mu|+k} \theta-\operatorname{dim} \nu / \mu P_{\nu}^{*}(x ; \theta) .
$$

Evaluation of (5.4) at $x=\lambda$ gives

$$
(|\lambda|-|\mu|) ! P_{\mu}^{*}(\lambda)=\theta-\operatorname{dim} \lambda / \mu P_{\lambda}^{*}(\lambda) .
$$

In particular, for $\mu=\emptyset$ one has

$$
|\lambda| !=\theta-\operatorname{dim} \lambda P_{\lambda}^{*}(\lambda) .
$$

Dividing (5.5) by (5.6) we obtain (5.2). 


\section{Integral representation of Jack polynomials}

Write $\nu \prec \lambda$ if

$$
\lambda_{1} \geq \nu_{1} \geq \lambda_{2} \geq \nu_{2} \geq \cdots \geq \nu_{n-1} \geq \lambda_{n}
$$

For any number $r$ set $(t)_{r}=\Gamma(t+r) / \Gamma(t)$. We have (see [M], VI.7.13')

$$
P_{\lambda}\left(x_{1}, \ldots, x_{n} ; \theta\right)=\sum_{\nu \prec \lambda} \psi_{\lambda / \nu} P_{\nu}\left(x_{1}, \ldots, x_{n-1}\right) x_{n}^{|\lambda / \nu|}
$$

where

$$
\psi_{\lambda / \nu}=
$$

$$
\prod_{i \leq j \leq n-1} \frac{\left(\nu_{i}-\lambda_{j+1}+\theta(j-i)+1\right)_{\theta-1}\left(\lambda_{i}-\nu_{j}+\theta(j-i)+1\right)_{\theta-1}}{\left(\lambda_{i}-\lambda_{j+1}+\theta(j-i)+1\right)_{\theta-1}\left(\nu_{i}-\nu_{j}+\theta(j-i)+1\right)_{\theta-1}} .
$$

Suppose $\mu$ has less than $n$ parts. The formulas (3.2) and (6.1) together imply

$$
P_{\mu}^{*}(\lambda)=\frac{(n \theta)_{\mu}}{((n-1) \theta)_{\mu}} \sum_{\nu \prec \lambda} \psi_{\lambda / \nu} \frac{P_{\nu}(1, \ldots, 1)}{P_{\lambda}(1, \ldots, 1)} P_{\mu}^{*}(\nu)
$$

where 1 is repeated $n$ times in the denominator and $(n-1)$ times in the numerator. Note that after substitution of (6.2) and of (3.1) rewritten as

$$
P_{\lambda}(1, \ldots, 1)=\prod_{i<j \leq n}\left(\lambda_{i}-\lambda_{j}+\theta(j-i)\right)_{\theta} \prod_{i \leq n} \Gamma(\theta) / \Gamma(\theta i)
$$

the formula (6.3) becomes the formula (7.16) from [Ok3]. We replace $\lambda$ and $\mu$ in (6.3) by their multiples $\kappa \lambda$ and $\kappa \mu$ and let $\kappa \rightarrow \infty$. By (2.3)

$$
P_{\mu}^{*}(\kappa \lambda) / \kappa^{|\mu|} \rightarrow P_{\mu}(\lambda), \quad \kappa \rightarrow \infty
$$

Introduce the following product of beta-functions

$$
C(\mu, n)=\prod_{i \leq n-1} B\left(\mu_{i}+(n-i) \theta, \theta\right)
$$

Set

$$
\Pi(\lambda, \nu ; \theta)=\prod_{i \leq j}\left(\lambda_{i}-\nu_{j}\right)^{\theta-1} \prod_{i>j}\left(\nu_{j}-\lambda_{i}\right)^{\theta-1} .
$$

Using the well known relation $(t)_{\theta} / t^{\theta} \rightarrow 1, t \rightarrow \infty$, one obtains from (6.3) the following 
Proposition. If $\mu$ has $<n$ parts then

$$
P_{\mu}(\lambda)=\frac{1}{C(\mu, n)} \frac{1}{V(\lambda)^{2 \theta-1}} \int_{\lambda_{2}}^{\lambda_{1}} d \nu_{1} \ldots \int_{\lambda_{n}}^{\lambda_{n-1}} d \nu_{n-1} P_{\mu}(\nu) V(\nu) \Pi(\lambda, \nu ; \theta) .
$$

Here $\lambda_{1} \geq \cdots \geq \lambda_{n}$ are arbitrary real (this assumption is not essential since $P_{\mu}$ is symmetric). If $\theta=1,2, \ldots$ then the integrand is holomorphic and $\lambda$ can be arbitrary complex. Iteration of this proposition together with (1.2) gives an integral representation for all Jack polynomials.

This proposition was first obtained by G. Olshanski (unpublished) for special values $\theta=1 / 2,1,2$ by using the group theoretic interpretation of the corresponding Jack polynomials. Then A. Okounkov [Ok3] found a general method which works for any $\theta$ and even for Macdonald polynomials. The proof presented above differs from that from [Ok3].

\section{Other applications}

The binomial theorem itself and the formula (5.2) result in an $\theta$-analog of the Vershik-Kerov theorems [VK1,VK2] about characters of $U(\infty)$ and $S(\infty)$. These results will be discussed elsewhere.

\section{References}

[Bi] C. Bingham, An identity involving partitional generalized binomial coefficients, J. Multivariate Anal. 4 (1974), 210-223.

[D] C. Dunkl, Hankel transforms associated to finite reflection groups, Hypergeometric functions on domains of positivity, Jack polynomials, and applications (Tampa, FL, 1991), Contemp. Math., vol. 138, 1992, pp. 123-138.

[DR] A. H. Dooley and J. W. Rice, On contractions of semisimple Lie groups, Trans. Amer. Math. Soc. 289 (1985), 185-202.

[FK] J. Faraut and A. Korányi, Analysis on symmetric cones, Oxford Univ. Press, 1994.

[J] M. F. E. de Jeu, The Dunkl transform, Invent. Math. 113 (1993), 147-162.

$[\mathrm{K}] \quad$ J. Kaneko, Selberg integrals and hypergeometric functions associated with Jack polynomials, SIAM J. Math. Anal. 24 (1993), 1086-1110..

[Kn] F. Knop, Symmetric and non-Symmetric quantum Capelli polynomials (to appear).

[KS] F. Knop and S. Sahi, Difference equations and symmetric polynomials defined by their zeros, Internat. Math. Res. Notices (1996), no. 10, 473-486.

[Lasc] A. Lascoux, Classes de Chern d'un produit tensoriel, C. R. Acad. Sci. Paris 286A (1978), 385-387.

[La] M. Lassalle, Une formule de binôme généralisée pour les polynômes de Jack, C. R. Acad. Sci. Paris, Sér. I 310 (1990), 253-256.

[M] I. G. Macdonald, Symmetric functions and Hall polynomials, second edition, Oxford Math. Monographs, Oxford University Press, New York, 1995.

[N] M. Nazarov, Yangians and Capelli identities, A. A. Kirillov, Seminar on representation theory; Amer. Math. Soc. Transl., q-alg/9601027 (to appear).

[Ok1] A. Okounkov, Quantum immanants and higher Capelli identities, Transform. Groups 1 (1996), no. 1, 99-126.

[Ok2] — Young basis, Wick formula, and higher Capelli identities, Internat. Math. Res. Notices (1996), no. 17, 817-839. 
[Ok3] _ (Shifted) Macdonald polynomials: q-integral representation and combinatorial formula, q-alg/9605013 (to appear).

[Ok4] _ Binomial formula for Macdonald polynomials, q-alg/9608021 (to appear).

[Ok5] Shifted Macdonald polynomials with 3 parameters and binomial formula for Koornwinder polynomials, q-alg/9611011 (to appear).

[OO] A. Okounkov and G. Olshanski, Shifted Schur functions, St. Petersburg Math. J., qalg/9605042 (to appear).

[OO2] Shifted Schur functions II, in A. A. Kirillov Seminar on Representation Theory, Amer. Math. Soc. Transl., q-alg/9612025 (to appear).

[Op] E. M. Opdam, Dunkl operators, Bessel functions, and the discriminant of a finite Coxeter group, Compos. Math. 85 (1993), 333-373.

[OV] G. Olshanski and A. Vershik, Ergodic unitarily invariant measures on the space of infinite Hermitian matrices, in Contemp. Math. Phys. F. A. Berezin's memorial volume (R. L. Dobrushin, R. A. Minlos, M. A. Shubin, A. M. Vershik, eds.), Amer. Math. Soc. Transl., Series 2, Vol. 175 (Adv. Math. Sci. - 31), Amer. Math. Soc., Providence, R.I., 1996, pp. 137-175.

[S1] S. Sahi, The spectrum of certain invariant differential operators associated to a Hermitian symmetric space, Lie theory and geometry: in honor of Bertram Kostant, Prog. Math. (J.-L. Brylinski, R. Brylinski, V. Guillemin, V. Kac, eds.), vol. 123, Birkhäuser, Boston, Basel, 1994.

[S2] - Interpolation, integrality, and a generalization of Macdonald's polynomials, Internat. Math. Res. Notices (1996), no. 10, 457-471.

[St] R. P. Stanley, Some combinatorial properties of Jack symmetric functions, Adv. Math. 77 (1989), 76-115.

[VK1] A. M. Vershik and S. V. Kerov, Asymptotic theory of characters of the infinite symmetric group, J. Funct. Anal. Appl. 15 (1981), 246-255.

[VK2] _ Characters and factor representations of the infinite unitary group, Soviet Math. Dokl. 26 (1982), 570-574.

Institute for Advanced Study, Princeton, NJ 08540

Current address: Deptartment of Mathematics, University of Chicago, 5734 South University Ave., Chicago, IL 60637-1546

E-mail address: okounkov@math.uchicago.edu

Institute for Problems of Information Transmission, Bolshoy Karetny 19, 101447 Moscow GSP-4, Russia

E-mail address: olsh@ippi.ac.msk.su 